\title{
SDGs and Geospatial Information Perspective From Nigeria-Africa
}

\author{
Muyiwa Agunbiade \\ University of Lagos, Nigeria \\ Olajide Oluwafemi \\ University of Lagos, Nigeria \\ Oluyemi Akindeju \\ University of Lagos, Nigeria
}

This chapter discusses SDGs connectivity by exploring the nature of interlinkages between the SDGs from the lens of geospatial information and geospatial data infrastructure. It also focuses on evolving an integrated framework towards achieving SDGs in developing economies.

\subsection{Introduction}

To develop further on the achievements of the MDGs and to complete what has not been achieved, the United Nations adopted, in 2015, the 2030 Agenda for sustainable development, setting 17 development goals and 169 targets. The world has witnessed tremendous progress in the living condition of many people, notwithstanding the challenges associated with the implementation of the MDG goals. This progress, however, was uneven. It was anticipated that Africa progress towards sustainable development will have implications on the global achievement. Paradoxically, while many countries in developed world achieved many of the goals and were able to monitor progress, many African countries, including Nigeria, did not make appreciable progress. Nigeria's record in achieving the MDGs has been abysmally low [5].

Like the preceding MDG goals, implementing the indivisible SDG agenda by policy actors is faced with challenges [3] especially regarding the nature 
of interlinkages between the SDGs. The complex nature associated with the indivisible characteristic of the 2030 Agenda also requires an unbundling. In other words, it is important to understand the possible trade-offs as well as synergistic relations between the different SDGs in a way that is enough to achieve long-lasting sustainable development results. Most importantly, in an era when communities and their various challenges are interconnected across different domains and jurisdictions. The implementation of SDGs is challenged by the required science-based analysis that is anchored on robust fit-forpurpose geospatial information. One of the long-standing issues, in this regard, is the lack of reliable data in appropriate format to aid decision-making and monitor progress. Where data are available, they are fragmented among different institutions and agencies (private and public), and as such not integrated and accessible. In addition, the available data are not disaggregated in a usable format. Specifically, it is established that accurate and reliable geospatial data, integrated across sectors, are central to the implementation and monitoring of progress towards the attainment of SDGs [14]. It is also established that geospatial data and urban processes interact in complex and integrated ways to foster sustainable development. While the application of geospatial technologies and Spatial Data Infrastructure in development processes is increasingly emerging globally, the regional spread has not been proportionate, with most developing countries lagging behind.

It has been recognised that the 169 targets, currently being further defined by the measurable indicators, are designed to help evaluate and monitor the implementation of SDGs and determine if the 17 goals are achieved. Indicators are important tools to support decision making process and measure what matters. Indicators are particularly necessary for the monitoring and evaluation of SDGs for quality, consistency and comparability of data over time and space and across sectors and regions [1]. However, Africa, in particular, faces a unique challenge of measuring the attainment of these goals due to the paucity of adequate and appropriate geospatial information that is in the right format and that is fit-for-purpose. This is further compounded by lack of interagency collaboration and uncoordinated policies. This lack of synergy between geospatial data infrastructure and SDGs and clear interactions within the SDGs is evident as there is increasing need for reliable baseline data to monitor and evaluate progress. This identified gap is a huge obstacle to many African countries in their quest for sustainable development [15]. Couple with this challenge, is the lack of clear road map for integration of geospatial data into national development and policy making process.

Most often, the baseline data are "set on the available information, which in many cases, is scanty, unreliable and dubious" ([19], p. 5). Considering this precarious situation, it is apparent that Geospatial information is a requisite prior to the monitoring phase of SDGS. This provides the necessary background to establish the prevailing condition [19]. Currently, there is a general lack of adequate geospatial information policy and governance due to the ineffective and inefficient institutional framework and tools. Africa is also 
challenged by issues around capacity and knowledge transfer. Yet, there is an increasing array of global challenges, including interregional issues such as peace, security, natural disasters and climate change that needs to be measured, which no nation or region can solve independently. Thus, the justification for calls regarding global coordination that is firmly anchored on good Geospatial Information and geospatial data infrastructure.

While geospatial information is essential, the production of geospatial data infrastructure domain across Africa is subject to particular institutional and knowledge constraints. There seems to be an enduring knowledge gaps between the supply and demand sides of data as demonstrated by the lack of institutional capacity to coordinate. As such, both supply and demand sides are problematic, and they are huge obstacles to development. In this regard, as illustrated by Jerven (2013), one of the challenges is the unstructured nature of interactions between producers and consumers [10]. There seems to be a lack of understanding on the nature of data and how such data should be collected, analysed, disaggregated and integrated for effective policy making and national development. Before now, Riddel (1990) observed that maybe the major challenge with the existing Africa data is that they are generally known to be erroneous, but the extent of inaccuracy cannot be easily ascertained [13]. Even today, nothing seems to have significantly changed in many of the African countries despite the steady improvement in geospatial data technologies globally and despite the urgent need for integrated data platform to support the complex interrelationship of the sustainable development agenda. As noted by International Council for Science (2017), the "SDGs interact with one another as an integrated set of global priorities and objectives that are fundamentally interdependent" [3]. Strategies to understand the range of positive and negative interactions among SDGs is critical to revealing their full potential at any scale.

However, there are different dimensions to the issues of connectivity between, geospatial information and SDGs. The central arguments are that: i) while the 17 SDG goals are broad, they are interdependent, and the success of the 2030 Agenda lies in demystifying the interlinkages between policy areas as presented in the sustainable development goals (SDGs). Meaning that to achieve all the 169 targets it will require, specifically, the development of Geospatial Information infrastructure, to help facilitate the connectivity. ii) It could also be argued that it is important to localize the SDGs by highlighting the role of local institutions and local actors. These are considered as key in achieving and contextualising separate list of targets for respective goals, thus, the perspective from Africa as a region is critical for fit-for-purpose analyses and solutions. In this regard, indicators must be consciously formulated to meet regional needs, aspirations and priorities. iii) the "leaving no one behind" principle of the SDGs that is intended to ensure, on the assumption that, a sustainable and smart future will develop a better future for all communities appears high-level and difficult to achieve in Africa. iv) the unavailability of effective and efficient land administration, that is, the lack of 
matured land information (LIS) and formal land registration systems, can be argued to be the root of most problems in African countries. Thereby incubating insecure land rights and social exclusion, economic regression, and environmental degradation.

This chapter starts by providing insights into the past events at implementing the MGDs, in Africa especially in Nigeria. This is based on the consideration that Nigeria's record in achieving the MDGs has been significantly low and the assessment of current SDGs' level of success or achievements will continually depend on the harmonization and utilization of a whole lot of tremendous data both spatial and non-spatial. As such, it requires the development of a platform like the geospatial data infrastructure. The next section reviews the existing knowledge and discusses the interconnection between the SDGs and geospatial information, taking into consideration: geospatial information, urban and rural resilience; approaches to integrating geospatial information and technologies in the implementation of the SDGs. Considering that all developments have spatial dimension, that is location-based, the key information required is geospatial information ([19], p. 5).

\subsection{Existing Knowledge About Interconnection Within SDGs and Between Geospatial Information}

This section provides a review of interdisciplinary analysis and multisectoral expertise on the usefulness and application of geospatial information. It reviews the interconnection between the SDGs and geospatial information while drawing inferences as regards the implications for urban and rural resilience. As noted by International Council of Science (2017), the underlying principle is that SDG goals interact with one another [3]. However, the "approaches for how to more systematically identify, characterize and address interactions between all sustainable development policy issues remains a challenge" ([9], p. $1499 ;[8])$.

\subsubsection{The Interconnection Between the SDGs, Geospatial Information, Urban and Rural Resilience}

As noted by Nilsson, et al. (2018), understanding both the negative and positive interactions between the SDGs is essential for decision-making that promotes the implementation of sustainable development[8]. In another words, the interactions across the SDGs should be thought through systematically. Nilsson, et al. (2018) provide insights into the mapping and assessing of SDGs interactions, using a defined typology and characterization approach, and summed that negative interactions are outnumbered by positive ones. This suggests that a more integrated policy making has the potential to provide 
more effective development outcomes. Nilsson, et al. (2018) further observed the challenges in identifying and assessing all the key interactions comprehensively at the global scale. They, therefore, argued for context-specific understandings. This argument is consistent with the overarching premise of the 2030 Agenda that underscores a nationally adapted interpretations and action on the SDGs ([8], p. 1499).

An essential component of determining integration is the consideration for the contextual meaning of the SDGs targets. There are nuance interpretations of the expectations as to the progress expected from each target. This simply means that, before assessing interactions, one needs to articulate what should be looked out for to articulate progress on a target, especially from the (subnational or national) context of implementation in terms of actual, observable outcomes ([8], p. 1499).

While it is essential to determine the linkages between different targets set for the SDGs, it is also important to consider that understanding the interdependent existence of people and the space they inhabit, is largely determined by the availability of geospatial data. One could infer, in this regard, that the level of connectivity at local, national, and global levels shapes future urban forms. Especially now that there is a noticeable intensification of challenges in our cities due largely to: increase in population growth and human mobility with majority of the world population now living in cities [4]; significant disasters that are weather related traceable to climate impact; limited resources to cope with the unintended consequences of population growth and related disasters. Significant part of this is linked to the lack of a comprehensive spatial data infrastructure, that has been argued to impede the process of strengthening community and infrastructure resilience, thus, preventing the protection of social and environmental sustainability, and narrowing the development gap, especially in the developing countries like Africa.

However, one of the key findings is that there is clearly no one-size-fits-all approach to understanding target interactions and infrastructure resilience. Noting that building on the existing knowledge will require a commitment to continuous iteration and improvement.

\subsubsection{Geospatial Information to Support Inclusive Urbani- sation, Resilient Development, and the SDGs}

The logical question to ask is that how does geospatial information supports and informs inclusive and even urbanisation, resilient development, and the SDGs? Earlier, Feeney et al. (2001) linked the increasing need to organize data across sectors and institutions, through the development of SDIs, to the growing need of addressing the complex and multiple challenges associated with sustainable development [2]. The primary aim for developing SDIs is to achieve better outcomes from spatially related development decision-making across economic, social and environmental spheres.

Despite the widespread adoption of digital technology and a high internet 
penetration rate (highest in Africa), existing databases are isolated, uncoordinated and lack harmony for a holistic policy framework for decision making. The isolation of digital information gathered by various agencies and department on individuals, spatial components and events provides a veritable start point to implement geospatial information framework for a localized connectivity of the global SDG agenda. The domestication of the global agenda is, therefore, necessary to set the stage for effective implementation and monitoring.

As noted by Scott \& Rajabifard (2017), the challenges of achieving sustainable development is not only about some sets of significant social, economic and environmental issues that are almost entirely geographic in nature [15]. Rather, it could also be noted that, geospatial information can provide a set of science and time-based monitoring solutions to these challenges, especially those that are driven by spatially enabled data.

Pesch (2014), posited that irrespective of rational interactions and connections over a long period of time, the reality is that there has been a limited connection and integration between sustainable development and geography, geospatial information and associated enabling infrastructure such as National Spatial Data Infrastructures (NSDIs) at both the technical and political levels [11]. This is not peculiar to developing nations but also highly data-rich and technology-driven nations. Wu Hongbo, UN Under-Secretary-General for Economic and Social Affairs, emphasized the role of geo-statistical data in improving governments' ability to 'examine, monitor, manage, propose and predict development and growth options for a sustainable future'. Wu also stressed the importance of geospatial information in decision making, policy formulation, measuring and monitoring development, including the post-2015 agenda.

As an all-encompassing, comprehensive global blueprint, the applicability of the 2030 Agenda in all countries, in all contexts, and at all times earns it the universality attribute as one of the core underpinning principles. Other principles include Leaving no one behind, Interconnectedness and Indivisibility, Inclusiveness and Multi-Stakeholder Partnerships. The geospatial community is strategically positioned to integrate geospatial information into the global development agenda, specifically in a way that will support measuring and monitoring the targets and indicators of the SDGs, with the core principles at the heart of it.

\subsubsection{Approaches to Integrating Geospatial Information and Technologies in the Implementation of the SDGs}

This section assesses the existing framework for a more holistic approach to integrating geospatial information and technologies in the implementation of the SDGs, by first reviewing the challenges for achieving the SDGs. Scott and Rajabifard (2017), out of serious concern for the attainment of SDGs, raised a fundamental question: How can geospatial information be implemented and integrated into national information systems, at a policy level, in order to 
contribute more holistically to measuring and monitoring the targets and indicators of the SDGs at a technical level? ([15], p. 60). This is, perhaps, against the realisation that while the evolution of sustainable development and the development of geospatial information have progressed in parallel period, frameworks for their integration have remained largely undeveloped even in developed countries [15]. In this regard, Pesch (2014) earlier notes that for a very long time, even in a highly data-rich and technology-driven global countries, there has been very little connection between sustainable development and geospatial information at either the political or the technical arena [11]. For example, while global leaders have committed much effort in developing targets and indicators to benchmark progress, there has been little understanding about the strategic direction for the integration of geospatial data information for efficient monitoring the implementation and achievements of the goals [15].

It is even paradoxical that while the United Nations - the proponent of SDGs - report on the 'Future We Want' acknowledged the value of reliable geospatial data for sustainable development [6], the report failed to clearly demonstrate strategies to mainstream geospatial data infrastructure into sustainable development [15]. For an effective integration, a clear integrated strategic direction, which takes into consideration national realities and regional peculiarities, is required. The challenges of developing such an integrated approach - a fit-for-purpose geospatial data framework - is proving difficult for many decision makers around the globe. This is, however, acute in developing countries, especially the African countries.

In recent times, however, with much international advocacy and dialogues coupled with the need for baseline data to monitor and evaluate progress towards sustainable development goals, the effort for integration is gaining momentum. The research efforts of the global geospatial community have provided useful frameworks, at both the policy and practical levels, to facilitate the integration of geospatial information and technologies in the implementation of the SDGs.

Scott and Rajabifard (2017); UN-Habitat (2016) works on sustainable development and geospatial information offer a useful strategic framework for integration which could serve as building blocks for implementation within the national geospatial strategic framework $[15,4]$.

\subsection{Framework and Methods}

This section adopts conceptual and empirical approaches for understanding contextual interactions between the SDGs targets, drawing on SDGs interactions framework as developed by Nilsson et al. (2018) [8]. The interactions depend on the meaning and the transparency of the assumptions associated 
with the interactions. One of the major benefits of this approach is the ability to critically and systematically navigate the several dimensions of the 2030 agenda, with particular focus on the contextual meaning of the targets. This is important to be able to establish the interactions between the targets. It should also be noted that policy and/or regulatory mandates also have the capability to affect the nature of interactions.

The central consideration for the framework revolves around the typology and scoring of interactions on a 7-point scale to identify causal and functional relations as it relates to the achievement of the sustainable development goals and targets [4]. In addition, key contextual determinants that impact on the interaction are governance and geographical contexts, implementation technologies, policies and time-horizon. This chapter adopts this interaction framework and emphasizes on: governance context, geographical context, spatial data infrastructure as embedded in implementing technology, and the time dimension.

With regards to the governance context, the assessment of the SDGs and targets are critically dependent on good governance. Inappropriate governance measure can potentially impact interactions to the extent that positive interactions can be reversed and turned into negative one. Regarding the geographical context, interactions is reinforced depending on where such interactions take place. Especially where cross-scale and cross-geographical interactions occurs.

Equally important is the 'implementing technology' that focuses on geospatial data/information. This framework has necessitated a need to set up and monitor policy level mechanism, leading to a harmonious integration of Geospatial and Statistical information for sustainable development in Africa [19]. Interaction is also impacted within the consideration of the time frame for the assessment. Therefore, articulating the trade-offs, synergies and spin-offs between the goals of the SDGs is important to unlocking their full potential. Thus, whatever the scale, it is an important consideration that progress made in some areas is not made at the expense of progress in others.

To expand the spatial data infrastructure as embedded in the 'implementing technology', it will be important to consider the 5ps Model. This involves the categorisation of SDG goals based on the five critical dimensions of: people, prosperity, planet, partnership and peace. As such, the geospatial information efforts shall, on the basis of defined spatial limitations or extent (LGAs, States, Geopolitical zones or Geographic regions) be gathered on the basis of the People's demographic characteristics, their economic and industrial prosperity, the state and manner of planet resource utilization, fostered partnership among stakeholders and entrenched peaceful co-existence in the society. The categorisation of the goals based on the 5ps model include: People (goals 1,2,4); Prosperity (goals 3,6,7,8,9, 11); Planet (goals 13, 14, 15); Partnership (goals 17, 12); Peace (goals 6, 10, 16). The next section is structured through this framework and model. 


\subsection{Findings and Analysis}

\subsubsection{The Interactions Between Various Elements of GIM and the SDGs in African Context}

Going by the mission statement of GI4SD, it is anticipated that "Africa produces and uses authoritative and evidence-based Geospatial Information for the attainment of its sustainable development goals and agenda 2063 objectives". The statement went further to provide insight into what is meant by authoritative and evidence-based GI as referring "to rigorously controlled best quality and "official" - consensus-based - GI, and its attribute of objective, logically-led and uncertainty-free or reduced source of decision making" ([19], p. 8). However, the reality is different from this expectation. There are few challenges preventing this from happening as anticipated.

The challenge is not only limited to the availability of reliable sources of GI, but also on 'access, quality, completeness, currency, availability of standardized metadata, interoperability of GI datasets, traceability of GI products, rights of data producers, liability of GI service providers, GI products and services pricing' ([8], p. 1490). In addition, it is also about systematically focusing on the means of implementation considering issues like finance, technology, capacity building, trade, policy coherence, partnerships, data, monitoring and accountability.

As documented by UNGGIM (2016), "efforts to build capacity in GIM in Africa over the past 20 years have been supply driven and have typically reflected the mandates of mostly external actors. Local, national and regional applications of GIM have continued to expand in scope and relevance, but without a strong demand-driven agenda for building capacity in GIM. The outcomes of such efforts will continue to fall short of their true potential" ([19], p. 37).

\subsubsection{Geospatial Information: Strengthening Community, Infrastructure, and Institutional Resilience}

The role of geospatial information is twofold, linking the 'where' component of SDGs and make challenges in various locations more visible and assisting with spatially tracking progress. The areas of monitoring and review, focusing on high quality, timely, reliable, and disaggregated data, including earth observation and geospatial information, was captured in the UN 2015 General Assembly text:

We will support developing countries, particularly African countries, LDCs (least developed countries), SIDS (small island developing States) and LLDCs (land-locked developing countries), in strengthening the capacity of national statistical offices and data systems to 
ensure access to high quality, timely, reliable and disaggregated data. We will promote transparent and accountable scaling-up of appropriate public-private cooperation to exploit the contribution to be made by a wide range of data, including earth observations and geospatial information, while ensuring national ownership in supporting and tracking progress [7].

It is very essential to integrate information systems at a national level that flow up into a regional and global level. The framework is a national bottomup approach. In developing countries, the use of data construct framework depends on institutional and architectural arrangements. As the world increasingly moves to rich data paradigm turning data into valuable information to support decision making, regarding development challenges, also requires change. Achieving SDGs requires the use of geospatial information to overcome challenges such as land rights, food production, disaster risk reduction, safe human settlements, and other social, economic, and environmental issues at local, national, and global levels.

As Nigeria reaches for the top global positions in the urbanization ranking in a couple of decades, the myriad of environmental, social, economic, sociocultural and infrastructural challenges consequent upon this growth trend widens the risk and vulnerability factors and stretches the resilient limits of the cities, thereby posing enormous threats to the actualization of the SDGs. The geographical complexities and the demographic dynamics/socio-cultural diversities of Nigeria portends a huge challenge for fostering implementation of the goals, and therefore requires a more unifying, inclusive and localized approach.

\subsubsection{The Role of Geospatial Data Infrastructures and Ser- vices in Achieving the SDGs in African Context}

Many of the challenges associated with sustainable development can be analyzed, modeled, and mapped within a geographic context [17]. However, while many of the challenges have spatial dimension, at the development policy making level, not much is understood concerning the role of spatial attributes in sustainable development processes $[12,15]$. In this regard, one of the most important questions in development community today, which requires evidence, is: "how can geospatial data infrastructures and services enhance the attainment of SDGs?"

In response to this question, the global geospatial community has focused discussion on the role and value of geospatial data for governance and development [12]. This research effort received a major boost, in recent years, with the global adoption of the Sustainable Development Goals and, coincidentally, considerable advancement in the level of awareness of geospatial technologies [15].

Sustainable Development Goals, in its conceptualisation, recognise the 
complex, interdependent, integrated and indivisible nature of the physical, economic and social systems, and the diverse associated challenges. Its 17 goals and 169 targets, though global, also recognise the need for a diverse range of quality, accessible, timely and reliable disaggregated data for monitoring and evaluating the implementation and achievements at national and regional levels.

The interconnected nature of SDGs and its multisectoral and multilevel implementation approach, for developments that leave nobody or situation behind, call for a holistic approach that integrates spatially enabled data platform into the national development policy framework. Geospatial Data Infrastructure has, therefore, emerged as a valuable platform that enhances access and sharing of geospatial information across sectors and inter-agencies as well as integration for evidence-based decision making and sustainable policy formulation [15]. In essence, geospatial data infrastructure can provide enabling and coherent capability and the needed unifying platform for multisectoral and inter-regional collaboration, consensus and evidence-based decisionmaking [15]. As argued by Feeney et al. (2001), the primary objectives of geospatial data infrastructure is to provide a platform for data coordination across disciplines and institutions for a better development outcome across space and time [2]. Scott and Rajabifard (2017) expressed similar view that the need for geospatial data infrastructure is essentially for achieving 'better outcomes from spatially related economic, social and environmental decisionmaking' ([15], p. 64).

Geospatial Data Infrastructure is, therefore, increasingly being embraced globally, though with regional variations, as the world gradually coming to terms with the need for an integrative framework for evaluation and monitoring development progress. For instance, in Europe, while policy actors are conscious of the need to standardize geospatial infrastructure in order to enhance data quality, many of the African countries are facing data fragmentation [12]. While it may not be totally accurate to argue that developing countries are data poor, lack of platform to bringing together the existing fragmented data might be a more possible argument. Geospatial data infrastructure offers such a unique opportunity to overcome this challenge, as it provides holistic and sustainable platform to bridge gaps between data, data providers and data users, as well as time and space, thereby enhancing the visibility of data to support policy and development decisions.

Geospatial data infrastructure progressively became one of the valuable components of the infrastructure required for socioeconomic prosperity, ecological management and liveability across levels of human settlements. The United Nations report on the establishment of UN-GGIM stressed the role of geospatial data infrastructure in shaping the formation and implementation of sustainable development programmes and polices [16]. The report of the United Nations Committee of Experts on Global Geospatial Information Management, at its second annual session, aptly captured the many roles geospatial 
data infrastructure can play in monitoring and evaluating the implementation and outcomes of sustainable development across sectors. It stated that:

Perhaps most importantly, there is a strong belief that geography provides the integrative framework necessary to support the requirements of multiple information communities in a timely and effective manner--providing the right data at the right time to the right place. The same geospatial content, repurposed, can support applications ranging from agricultural management, to emergency planning and response, to scientific collaboration on climate change, to transportation planning. All of these applications have implications for sustainable development and liveability [17].

The absence of National Geospatial Data Infrastructure platform means that governments, at all levels, will rely on unstandardized and fragmented data for decision making. This is the situation in the developing countries, especially African countries, where governments are continually challenged with lack of timely data compounded with poor data quality and a general lack of interoperability between different sources of data $[15,18]$. One of the immediate consequences of this is that development decisions are based on inaccurate data. Data have the power to mislead or inform development and policy making. In any context, the capability to implement either national standards or globally agreed goals, such as SDGs, largely depends on the quality of available resources and data. For many reasons, the current data use for development and policy making in many African countries provide little guide for effective development. In general, institutional and structural characteristics of many Africa nations pose huge barriers in collecting and evaluating data to implement and monitor both local and global development goals.

Data essentially influence what is known about the state of development and subsequently shape decision making process. The United Nations report on the achievements of MDGs provided some reflections on the challenges and lessons learned from the implementation of MDGs. It recognises the global achievements of MDGs. It however acknowledges that reliable and timely data in appropriate format, an essential component of any development programme, to effectively prioritise policy agenda and monitor progress were inadequate in the implementation of MDGs. This is particularly acute in Africa. Commenting on these reflections, Scott and Rajabifard (2017) concluded that the implication was that MDGs were largely implemented in many developing regions, especially Africa, without reliable data or a sustainable data platform to aid consistent measure and monitor of implementation progress towards sustainable development [15].

Essential for global agenda, such as SDGs, that seeks to address complex and multiple challenges, is the need to have an adequate understanding of the interrelationship of the challenges in relation to space, time and people. 
Equally important is the ability to monitor trends of events, provide timely information, particularly to the population at risk, and prioritise responses and actions. Geospatial data infrastructure can help in these regards, as asserts by United Nations (2015) [7]:

Knowing where people and things are and their relationship to each other is essential for informed decision-making. Comprehensive location-based information is helping governments to develop strategic priorities, make decisions, and measure and monitor outcomes. Once the geospatial data are created, they can be used many times to support a multiplicity of applications.

One of such areas of applications is the possibility to link ecological and socioeconomic data in a way that clearly presents interconnections across the spheres of sustainable development - environment, economic and social - and how they influence one another. Geospatial data infrastructure provides such a unique opportunity to integrate geospatial data into national development framework in a more holistic and sustainable way. Considering the peculiarity of each geographical region and location in terms of environmental configuration and level of development, geospatial data, with standardised indicators as the object of measurement, provide necessary transparency and accountability for development governance and evidenced for policy making. Meeting the numerous goals of sustainable development requires the integration of geospatial data infrastructure platform.

Geospatial data infrastructure is essential for enhancing the political and social engagement of hitherto marginalised people and to shape policy and development outcomes through evidence. Though well-intended, it is proving difficult to be integrated into development framework in many developing countries. Geospatial Data Infrastructure, as an evolving platform, is much more than just data. Achieving SDGs requires conscious and evidence based spatial and socioeconomic decision making. SDI will play a pivotal role in enhancing the efficiency and adequacy of such decision. As argued earlier, the challenge is not limited to the availability of reliable sources of GI, but also on unrestricted access, completeness, currency, quality, availability of standardized metadata, provenance, interoperability of GI datasets, GI products and services pricing.

\subsection{Inferences, Future Direction and Conclusion}

As noted by UN-GGIM (2016), to achieve the UN-SDGs and AU Agenda 2063 targets, at national, sub-regional and regional levels in Africa, will require good governance and sound policies in Geospatial Information Management 
([19], p. 24). As discussed in the preceding sections, these will guide the way African countries, will get organised and operate in order to achieve maximum benefits of GIM efforts. In addition, leveraging the already initiatives such as UN-GGIM, UN-Expert Group on Land Administration and Management, UN-GGIM Private Sector Network and UN-GGIM Geospatial Societies will facilitate the harmonisation and standardisation of data and integration of multi-domain analytics.

Griggs et al. (2017), assert that "the process of systematically identifying and scoring interactions across the 17 SDGS using a common terminology is very valuable" ([3], p. 8). Consistent with this position, one could argue in support of Griggs et al. (2017) that the process allows broad multi-disciplinary and multisectoral conversations [3]. It also allows synthesis and scoping of knowledge needs while providing rational and concrete clustering of targets that need to be addressed together to allow integrated approach for implementation and monitoring. The major limitations, however, is the challenge relating to selecting the important interactions from all the possible alternatives, especially considering the different expert's characterisation of interaction. That is, the contextual meaning of each of the possible interactions.

In conclusion, geospatial information policy is required to effectively manage geospatial information for sustainable development. It should be seen as a compulsory requirement that is anchored on legal and coherent institutional environment, that is set to achieving the most cost-effective and fulfilling impact. Therefore, GI applications should not only be encouraged, but should be layered on political and institutional structure designs to strengthen transparency in governance. A strong political will, built on strong GI policy has capacity to produce good governance that respect objective, fair and equitydriven decision making.

\section{Bibliography}

[1] Daniel Lage Chang, Jamile Sabatini-Marques, Eduardo Moreira Da Costa, Paulo Mauricio Selig, and Tan Yigitcanlar. Knowledge-based, smart and sustainable cities: a provocation for a conceptual framework. Journal of Open Innovation: Technology, Market, and Complexity, 4(1):5, 2018.

[2] M Feeney, Abbas Rajabifard, and Ian P Williamson. Spatial data infrastructure frameworks to support decision-making for sustainable development. 2001.

[3] DJ Griggs, Mans Nilsson, A Stevance, David McCollum, et al. A guide to SDG interactions: from science to implementation. International Council for Science, Paris, 2017. 
[4] UN Habitat. Urbanization and development: emerging futures. World cities report, 3(4):4-51, 2016.

[5] Nigeria MDGs. Millennium development goals end point report, 2015, 2015.

[6] United Nations. The future we want. United Nations General Assembly. Resolution 66/288, 2012.

[7] United Nations. Transforming our world: the 2030 agenda for sustainable development. United Nations General Assembly. Resolution 70/1, 2015.

[8] Måns Nilsson, Elinor Chisholm, David Griggs, Philippa HowdenChapman, David McCollum, Peter Messerli, Barbara Neumann, AnneSophie Stevance, Martin Visbeck, and Mark Stafford-Smith. Mapping interactions between the sustainable development goals: lessons learned and ways forward. Sustainability science, 13(6):1489-1503, 2018.

[9] Måns Nilsson, Dave Griggs, and Martin Visbeck. Policy: map the interactions between sustainable development goals. Nature News, 534(7607):320, 2016.

[10] Poor Numbers. How we are misled by african development statistics and what to do about it, 2013.

[11] Udo Pesch. Sustainable development and institutional boundaries. Journal of Integrative Environmental Sciences, 11(1):39-54, 2014.

[12] Bhanu Rekha. Geospatial data: Key to achieve sdgs, 2016. Geospatial World.

[13] Roger Riddell. Manufacturing Africa: performance $\&$ prospects of seven countries in sub-Saharan Africa. James Currey Publishers, 1990.

[14] Michael Rigby and Serryn Eagleson. Spatial data infrastructure challenges for addressing the sustainable development goals in australia. 09 2018.

[15] Greg Scott and Abbas Rajabifard. Sustainable development and geospatial information: a strategic framework for integrating a global policy agenda into national geospatial capabilities. Geo-spatial Information Science, 20(2):59-76, 2017.

[16] UN-GGIM. Report of the secretary general on global geospatial information management. United Nations General Assembly E/2011/89, 2011.

[17] UN-GGIM. Monitoring sustainable development: Contribution of geospatial information to the rio +20 processes, 2012.

[18] UN-GGIM. Statement of shared guiding principles for geospatial information management, 2015. 
[19] UN-GGIM. Geospatial information for sustainable development in africa (gi4sd) african action plan on global geospatial information management (aap-ggim) 2016-2030. United Nations Economic and Social Council, E/ECA/GGIM/16/1, 2016. 